\title{
An Investigation of the Mechanisms of Stall Margin Improvement in Casing Treatment with Bridge Structure
}

\author{
Shuchao Huang ${ }^{1,2, a}$, Wei Yuan ${ }^{1,2, b}$ and Le $\operatorname{Han}^{1,2, c}$ \\ ${ }^{1}$ National Key Laboratory of Science and Technology on Aero-Engines Aero-thermodynamics, \\ School of Energy and Power Engineering, Beihang University, Beijing 100191, China; \\ ${ }^{2}$ Collaborative Innovation Center for Advanced Aero-Engine, Beijing 100191, China \\ ahscbuaa@163.com, byuanwei@buaa.edu.cn, chanle@buaa.edu.cn
}

\begin{abstract}
In this paper, casing treatment with a bridge structure is numerically investigated to study the mechanism of stall margin improvement. The results show that both the tip leakage flow and separation flow on the suction side of the blade can block the rotor tip passage and thereby lead to stall. Tip leakage flow is the main reason for stall in a compressor with a smooth shroud. Bridge casing treatment can control both the tip leakage flow and separation flow to balance stall margin improvement and efficiency loss by varying the suction area and the jet area. The results show that the enlargement of the jet area increases the attack angle; consequently, the separation flow increases on the suction side whereas the efficiency decreases. Moreover, it is observed that tip leakage decreases and stall margin increases with an increase in the jet suction area.
\end{abstract}

Keywords: Stall margin improvement; Stall mechanism; Casing treatment with bridge structure; Jet area; Suction area.

\section{Introduction}

In a transonic compressor, the low-speed area created by the interaction between the tip leakage vortex and the shock blocking the passage is considered an important factor leading to compressor stall [1-4]. Casing treatment is a method that significantly improves the performance of a compressor/fan, and it has been developed into many forms since Koch C C [5-9] found that it can improve the compressor stability margin. The traditional casing treatment includes axial slots, circumferential grooves, and chordwise grooves [10-16]. Greitzer [10] concluded that the traditional casing treatment can extend the stall margin at the expense of efficiency loss.

Osborn [11] found that an axial straight slot increases the stall margin by $15.8 \%$ and decreases the efficiency by $6 \%$ at a $90 \%$ design speed. Moreover, an axial skewed slot increases the stall margin by $15.3 \%$ and decreases the efficiency by $5 \%$ at a $45 \%$ design speed.

Prince [12] found that the axial skewed slot increases the compressor stall margin by $15.3 \%$ with a $1.5 \%$ reduction in peak efficiency $(\mathrm{PE})$, whereas a chordwise groove increases the compressor stall margin by $15 \%$ and reduces the PE by $1.4 \%$.

Müller [13] used circumferential grooves on the rotor of a single-stage transonic compressor. The results showed that circumferential grooves improve the compressor stall margin and reduce the efficiency by less than $0.4 \%$.

XianGjun Li [14] placed a shallow circumferential groove at different axial positions on a highly loaded transonic axial-flow compressor and found that it effectively increased the stability of the compressor and slightly increased the pressure ratio with an efficiency loss of less than $1 \%$.

XinGen Lu [15] used an axial slot and a circumferential groove on a single-stage transonic compressor and found that they increased the compressor stall margin by $23.6 \%$ and $5.06 \%$, with a concurrent adiabatic efficiency loss of $5.01 \%$ and $0.54 \%$, respectively.

Considering both the efficiency and the stall margin, Hathaway [16] proposed a self-recirculating casing treatment that, unlike the conventional casing treatment, has an external airflow path isolated from the compressor main flow. The downstream high pressure is sucked by the slot and then driven by the differential pressure before being ejected from the leading edge of the blade through the 
external airflow channel. The results show that this self-recirculating casing treatment does not affect the efficiency when the compressor margin increases.

The experimental results show that compared with the conventional casing treatment, the arc-skewed slot casing treatment (ASCT) designed by YaJun LU [17, 18] remarkably improves the stall margin without significantly affecting the compressor efficiency.

In the present study, to reduce the efficiency loss resulting from casing treatment, a bridge-type structure is designed based on the arc-skewed slot casing treatment. The design of this structure is inspired by the structural characteristics of the self-recirculating casing treatment. In the proposed system, a low-energy fluid is drawn from the rear end of the channel. It then exits from the leading edge of the blade, but there is no flow-interchange in the middle part of the slot because of the isolation of the bridge.

Furthermore, the proposed casing treatment with a bridge structure (CTBS) is numerically investigated for a compressor to study the mechanism of stall margin improvement by analyzing different suction areas and jet areas. To this end, the first stage of a transonic compressor is selected as the research object.

\section{Model and Numerical Methods}

Figures 1 and 2 show the difference between ASCT and CTBS. CTBS comprises the following three parts: the suction area, bridge channel, and jet area. The fluid enters the suction area from the high-pressure area at the rear end of the flow channel, goes through the bridge channel, and flows into the main stream via the jet area. The parameter $\lambda$, which represents the overlap percentage between the slot and the tip axial chord length, is defined as following:

$$
\lambda=C_{e} / L
$$
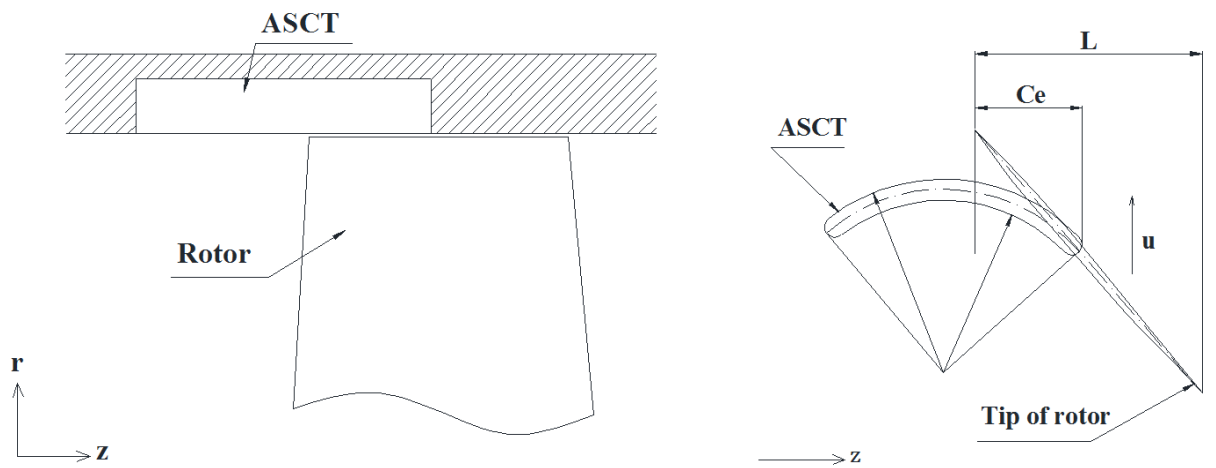

Fig. 1 Arc-skewed slot casing treatment (ASCT)
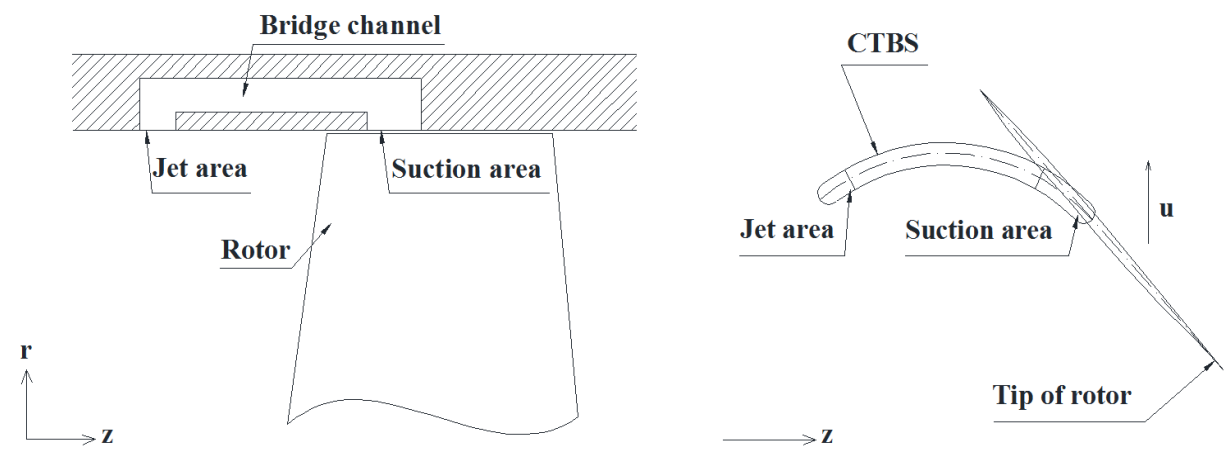

Fig. 2 Casing treatment with bridge structure (CTBS)

In this study, transonic compressor rotors with different walls were numerically simulated. One compressor had a smooth wall (SW, Fig.1), whereas another comprised CTBS (Fig.2). The number of rotor blades was 37, the design speed was $12036 \mathrm{rpm}$, and the tip relative Mach number was approximately 1.3. The number of bridge slots was 111 , which was thrice the number of rotor blades 
used herein. The overlap $\lambda$ was $45 \%$. Figure 3 shows the rotor blade mesh. Figure 4 shows a grid of CTBS. A transition layer mesh was set between the casing treatment and the shroud.

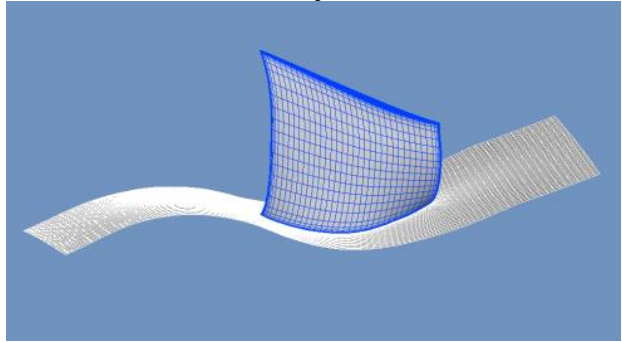

Fig. 3 Mesh of rotor

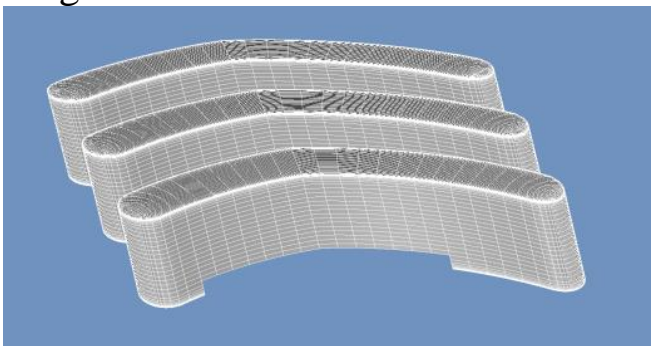

Fig. 4 Mesh of CTBS

The Reynolds-averaged Navier-Stokes equations were solved using the commercially available software ANSYS-CFX. The shear stress transport two-equation model was chosen as the turbulence model. To meet the requirements of the turbulence model, the distance of the first layer grid from the wall was adjusted such that Yplus was less than 1.

The simulation was performed at the design speed, the inlet boundary condition was set according to the standard atmospheric conditions, and the inlet direction was axial. The boundary condition of the outlet satisfied the radial equilibrium equation, and different working conditions could be obtained by adjusting the outlet static pressure. The bottom surface of the transition layer mesh and the top surface of the tip clearance mesh were set to the rotation-static interface condition. In addition, the frozen rotor method was used for the steady calculation.

\section{Results and Discussion}

\subsection{Analysis of the Stall Mechanism in the Compressor with a Smooth Wall}

Figure 5 shows the performance of the compressor with an SW. The PE is $90.856 \%$, the maximum pressure ratio at near stall (NS) is 1.9569 , and the stall margin is $10.54 \%$. Stall margin is defined as follows:

$$
\phi=\left(\frac{\pi_{n s}^{*} / \dot{m}_{n s}}{\pi_{p e}^{*} / \dot{m}_{p e}}-1\right) \times 100 \%,
$$

Where $\pi_{p e}^{*}$ and $\pi_{n s}^{*}$ are the total pressure ratios corresponding to the design point and NS point, respectively, and $\dot{m_{p e}}$ and $\dot{m_{p e}}$ are the mass-flow rates corresponding to the design point and NS point, respectively.

As shown in Fig.6, the Mach number of the blade tip is approximately 1.3. From the blade structure, it can be seen that the tip blade camber is curved. A curved camber indicates that the increasing pressure results from both the front shock and the rear divergent passage. Therefore, separation flow on the suction side of the blade may exist when the backpressure is sufficiently high. Both the tip leakage flow and separation flow on the suction side of the blade can block the rotor tip passage. With increasing backpressure and pressure ratio, the shock position of the suction surface gradually moves forward, as does the influence range of the tip leakage. Meanwhile, the separation-flow range on the suction surface also increases gradually.

Figure 7 shows the leakage flow at the tip near the stall point. It can be seen that the leakage flow crosses the shock wave and then blocks the tip channel. Comparing the passage block caused by tip leakage flow with that caused by the separation flow on the suction side, we conclude that stall is mainly induced by the leakage-flow block at the tip passage. 


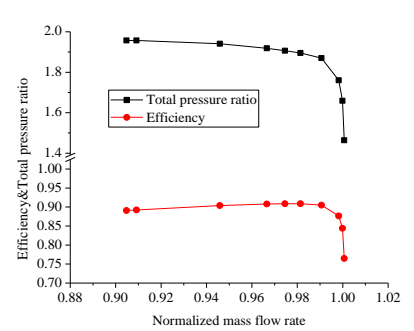

Fig. 5 Performance of the compressor with a smooth wall (SW)

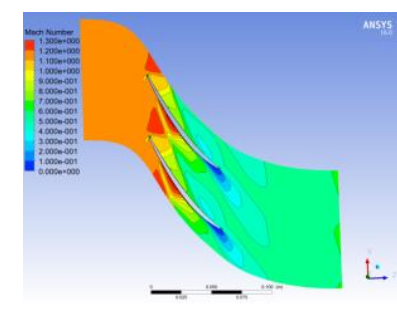

(a) Peak efficiency (PE)

Fig. 6 Relative Mach number contour near the tip of the rotor, SW

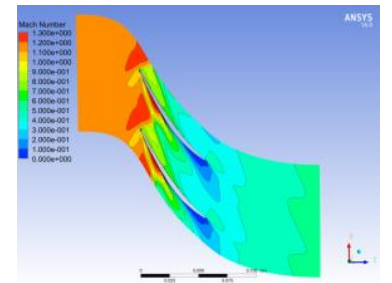

(b) Near stall (NS)

Fig. 7 Tip leakage flow of the rotor, NS

\subsection{Analysis of the Casing Treatment with a Bridge Structure (CTBS) Mechanism}

Table 1 lists the CTBS with different suction and jet areas. The percentage represents the ratio between the suction area (and the jet area) and the arc-skewed slot area. The variables are controlled to study the effects of different suction/jet areas on the stability of CTBS by only changing the jet area or the suction area. CTBS 1,2,3, and 4 maintain the same suction area at different jet areas, whereas CTBS 3, 5, and 6 maintain the same jet areas at varying suction areas.

Table 1 Different jet and suction areas for CTBS

\begin{tabular}{ccccccc}
\hline & CTBS 1 & CTBS 2 & CTBS 3 & CTBS 4 & CTBS 5 & CTBS 6 \\
\hline Jet area/\% & 5 & 8 & 13 & 21 & 13 & 13 \\
Suction area/\% & 25 & 25 & 25 & 25 & 11 & 18 \\
\hline
\end{tabular}

\subsubsection{Effect of Jet Area}

Figure 8 shows the flow-efficiency of the compressor with different jet areas under the same suction area. It can be seen from Table 2 that with increasing jet area, the efficiency gradually reduces. When the jet area percentage is $21 \%$, the maximum efficiency loss is $1.741 \%$. When the jet area percentage is $5 \%$, the efficiency loss is $0.52 \%$. The stall margin increases first and then decreases with increasing jet area; however, it does not change significantly after the jet area percentage exceeds $8 \%$. The stall margin of the CTBS compressor is 5\%-6\% higher than that obtained with the compressor with a SW.

Table 2 Compressor-performance parameters of CTBS for different jet areas

\begin{tabular}{ccccc}
\hline & Jet area/\% & Suction area/\% & PE/\% & $\phi_{/ \%}$ \\
\hline SW & & & 90.856 & 10.54 \\
CTBS 1 & 5 & 25 & 90.336 & 14.45 \\
CTBS 2 & 8 & 25 & 89.971 & 15.84 \\
CTBS 3 & 13 & 25 & 89.562 & 16.42 \\
CTBS 4 & 21 & 25 & 89.115 & 15.50 \\
\hline
\end{tabular}

Figure 9 shows the relationship between the jet area and the suction flow rate of CTBS. The ordinate represents the ratio of the suction flow rate to the compressor maximal flow rate. It can be seen from this figure that the suction flow rate near the NS point is higher than that at the PE point in the same jet area. Simultaneously, at the PE point and NS point, the suction flow rate of the CTBS compressor increases with the jet area.

Figure 10 shows the tip leakage flow of CTBS with different jet areas at the same flow rate as that at the NS point of the SW. With increasing jet area, the leakage flow gradually changes from flowing smoothly out of the main channel to crossing two different blades blocking the rotor tip passage. 


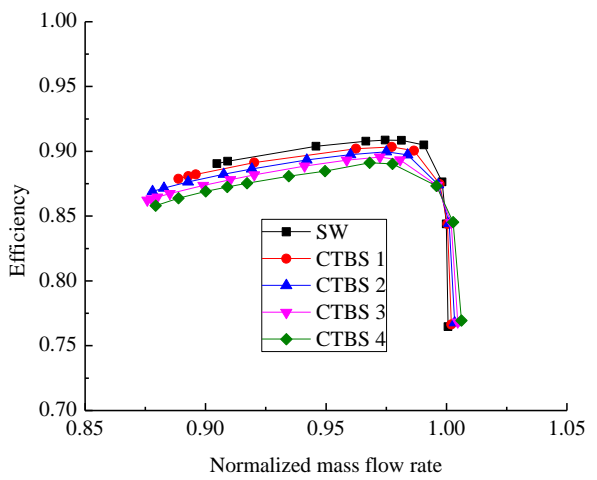

Fig. 8 Performance of the compressor with CTBS under different jet areas

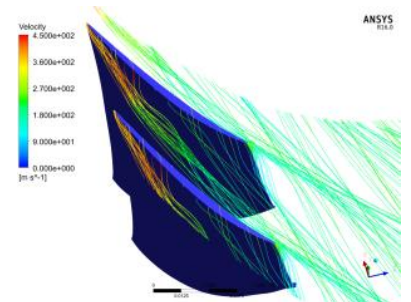

(a) CTBS 1

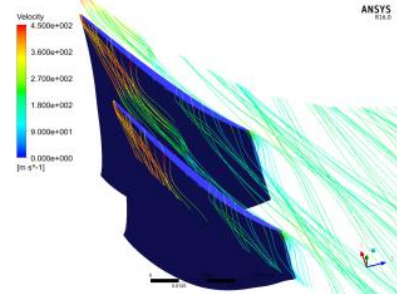

(b) CTBS 2

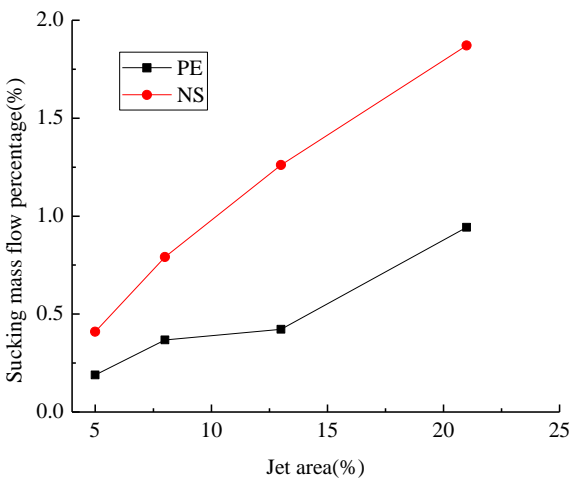

Fig. 9 Suction mass-flow percentage of the compressor with CTBS under different jet areas

Fig. 10 Tip leakage flow of the rotor with CTBS (same flow rate as that at the NS point of the SW)

Figure 11 compares the entropy increase near the tip of the rotor for three different jet areas. As the jet area increases from CTBS 2 to CTBS 3 to CTBS 4, the suction flow rate and the mixing loss between the jet flow and the mainstream increases. Thus, the entropy and efficiency loss increase.

Figure 12 shows the effect of inlet relative velocities and angles of attack on different jet areas; Wt represents the relative circumferential velocity, $\mathrm{Wz}$ is the axial velocity, and Beta refers to the angle between the relative velocity and the axial direction, thereby indicating the size of the attack angle. Near the tip position, the jet area increases with the jet flow rate and the relative circumferential velocity. Conversely, the smaller the axial velocity, the higher the angle of attack. However, for a spanwise under $95 \%$ height, the jet area increases with the axial velocity but decreases with the angle of attack. In other words, the larger the jet area, the smaller the flow capacity near the rotor tip and the greater the flow capacity under the $95 \%$ height.

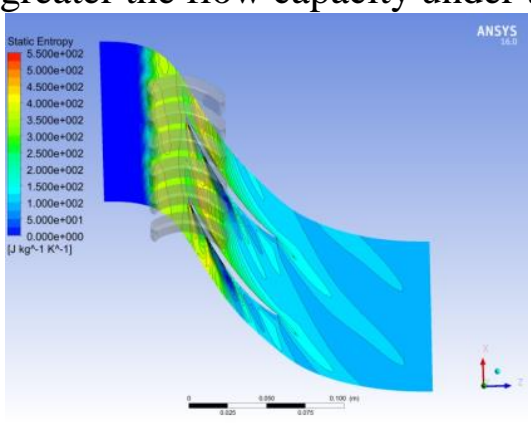

(a) CTBS 2

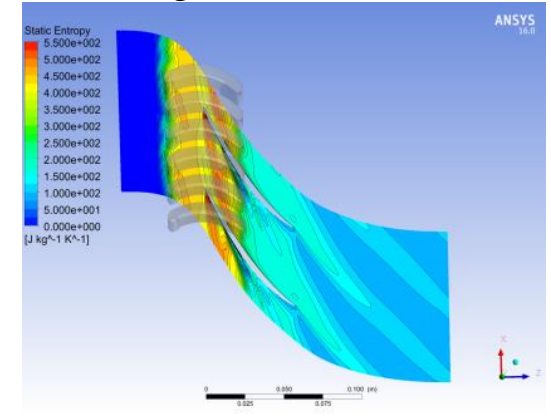

(b) CTBS 3

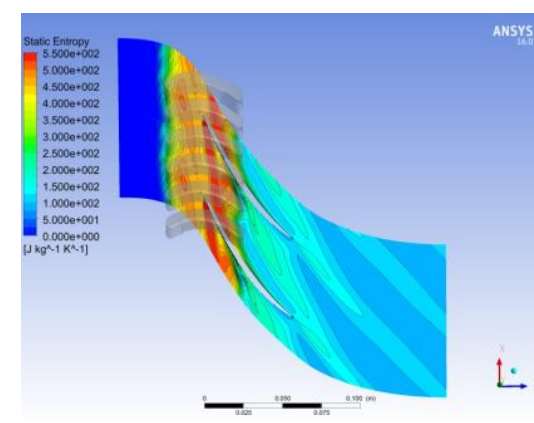

(c) CTBS 4

Fig. 11 Static entropy near the rotor tip with CTBS under different jet areas (same flow rate as that at the NS point of the SW) 


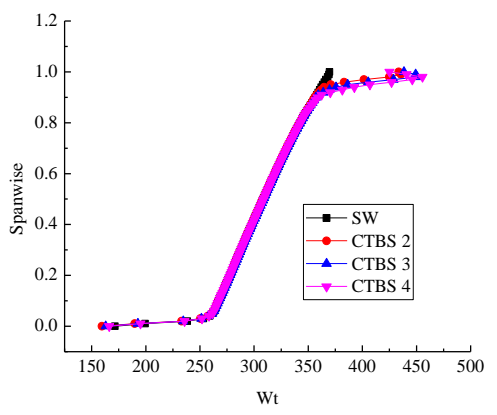

(a) $\mathrm{Wt}$

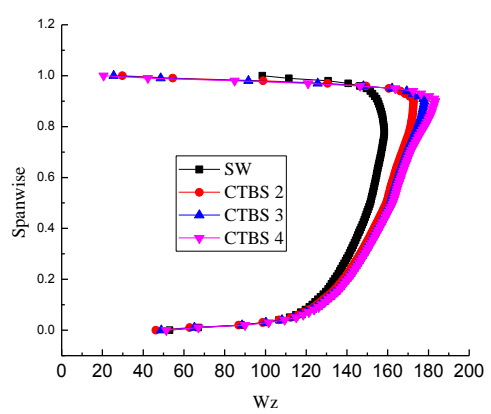

(b) $\mathrm{Wz}$

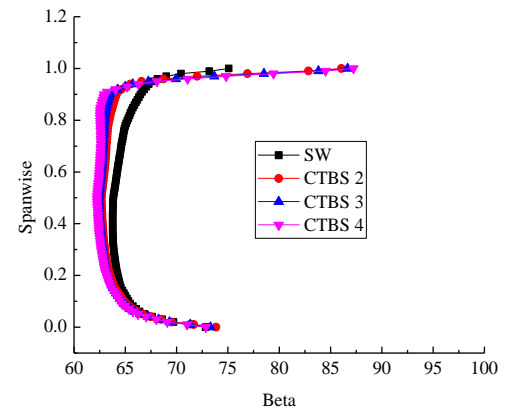

(c) Beta

Fig. 12 (a) Relative circumferential velocity (Wt), (b) axial velocity (Wz), and (c) the angle between the relative velocity and the axial direction of the rotor with CTBS (same flow rate that at the NS point of the smooth-wall)

Figure 13 shows the deviation angle along the tip blade suction surface at PE; this angle can indicate the separation on the suction side of the blade. With increasing jet area, the separation point of the blade gradually moves forward and the separation range increases. From the abovementioned analysis, we infer that the larger the jet area, the larger the angle of attack of the blade near the tip; this inevitably leads to an increase in the separation area on the suction surface. Meanwhile, an increase in the suction flow rate reduces the kinetic energy of the fluid near the tip blade passage under the rear part of the slot and weakens the ability of the tip fluid to resist the negative pressure gradient; consequently, the separation becomes more serious.

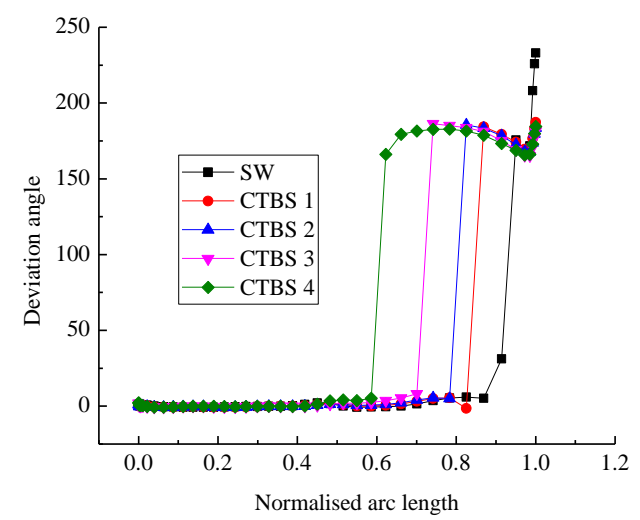

Fig. 13 Inner-flow deviation angle along the suction face near the rotor tip with CTBS under different jet areas

According to the present analysis results, under a constant suction area, the growth in the jet area increases the suction flow rate of the CTBS compressor and the attack angle of the blade near the tip. Thus, the separation area on the suction side increases and a more serious blockage occurs in the passage; this leads to a low PE.

\subsubsection{Effect of Suction Area}

Considering that CTBS 3 exhibits the largest stall margin improvement for a $13 \%$ jet area, in this section, we analyze the effect of CTBS for a $13 \%$ jet area.

Figure 14 shows the compressor-characteristic performance of CTBS under different suction areas when the jet area is kept constant. As the suction area increases, the stall margin of the compressor gradually increases and the change in efficiency is small. When the suction area is $25 \%$ (CTBS 3), the stall margin is the largest.

Figure 15 shows that with CTBS at the PE point and NS point, variations in the suction area have little effect on the suction flow rate of the compressor. Therefore, the suction capacity of CTBS is mainly determined by the jet area. Figure 16 shows the influence of separation on the suction surface under different suction areas. The separation is not affected by the changes in the suction area because the suction flow rate of CTBS does not change significantly under these conditions. 
Table 3 Performance-parameters of CTBS under different suction areas

\begin{tabular}{ccccc}
\hline & Jet area/\% & Suction area/\% & PE/\% & $\phi / \%$ \\
\hline SW & & & 90.856 & 10.54 \\
CTBS 5 & 13 & 11 & 89.849 & 13.04 \\
CTBS 6 & 13 & 18 & 89.618 & 15.19 \\
CTBS 3 & 13 & 25 & 89.562 & 16.42 \\
\hline
\end{tabular}

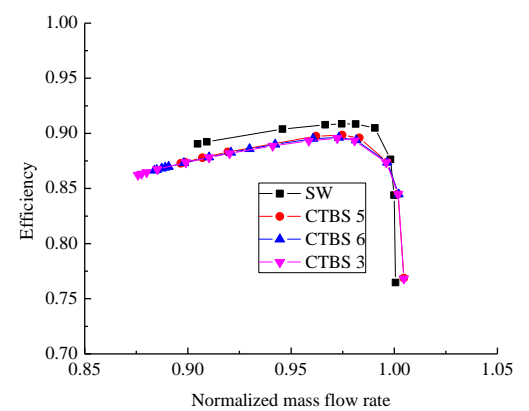

Fig. 14 Performance of the compressor with CTBS under different suction areas

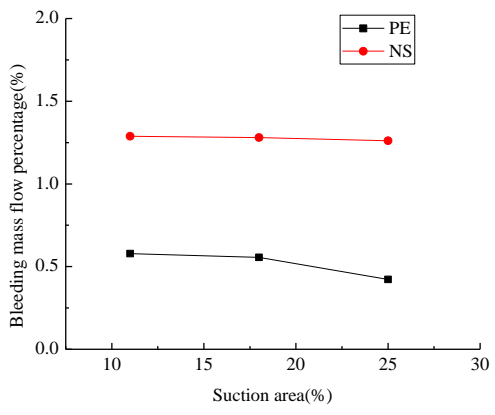

Fig. 15 Suction mass-flow percentage of the compressor with CTBS under different suction areas

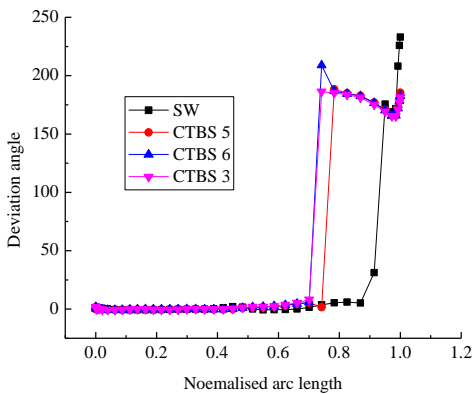

Fig. 16 Inner-flow deviation angle along the suction face near the rotor tip with CTBS under different section areas

Figure 17 shows the tip relative Mach numbers of CTBS 5, CTBS 6, and CTBS 3 at the same flow rate as that at the CTBS 5 NS point. In CTBS 5 (Fig. 17(a)), the tip passage blockage near the CTBS 5 NS point concentrates in two areas. One is the low-energy block area caused by the interaction between the tip leakage flow and the shock, and the other is the separation on the suction side. These two factors together block the tip passage and further lead to compressor stall. With increasing suction area (Fig. 17 (a) (b) (c)), the separation in the suction side does not change significantly whereas the block caused by tip leakage flow is gradually reduced. This analysis indicates that because of the reduced leakage flow, the stall margin improves as the suction area increases.

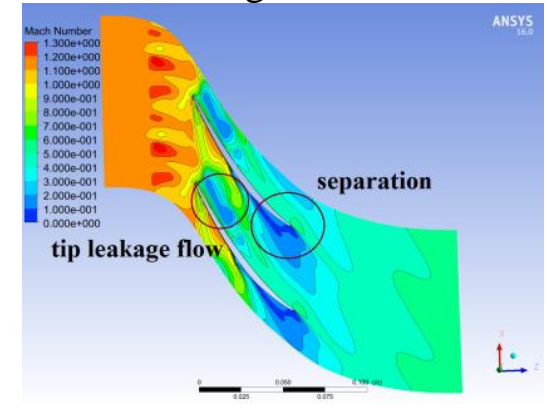

(a) CTBS 5

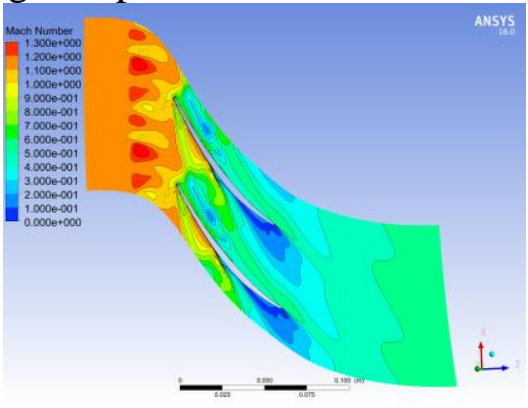

(b) CTBS 6

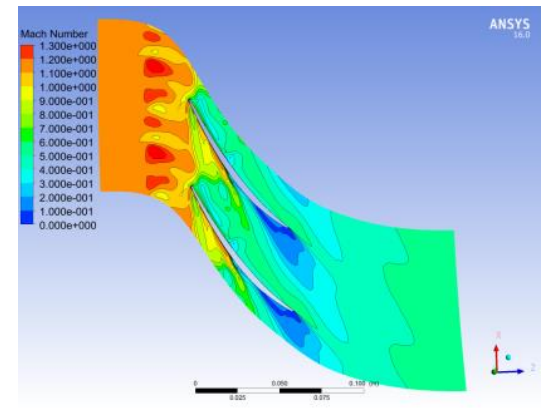

(c) CTBS 3

Fig. 17 Relative Mach number contour near the tip of the rotor (same flow rate as that for CTBS 5 at the NS point)

As per the abovementioned analysis, we infer that the suction area of CTBS mainly affects the stall margin of the compressor. An increase in the suction area creates a large stall margin. Compared with the jet area, changes in the suction area have little effect on the suction flow rate. Therefore, suction area has little influence on the separation in the suction side and does not significantly affect the efficiency.

\section{Conclusion}

In this paper, a transonic compressor with a Mach number of 1.3 was numerically simulated. We analyzed the stall margin improvement in this compressor using CTBS. The main results obtained by us are as follows: 
(1) The tip blade camber is curved for a Mach number of 1.3, such that both the tip leakage flow and separation flow in the suction side can cause a tip block; this leads to compressor stall. CTBS can control the tip leakage flow and separation flow to balance the margin improvement and efficiency loss by changing the size of the suction area and the jet area.

(2) At a constant CTBS suction area, an increase in the jet area produces a large suction flow rate, a large angle of attack near the tip, a large separation in the suction side, and serious blockage in the passage; this lowers the PE. Simultaneously, the tip leakage flow becomes more oblique and blocks in front of the blades.

(3) The CTBS suction area mainly affects the stall margin of the compressor. A large suction area produces a large stall margin. Compared with the jet area, variations in the suction area have little effect on the suction flow rate. Therefore, suction area has little influence on the separation in the suction side as well as the efficiency.

\section{Acknowledgements}

This investigation was supported by the Fundamental Research Fund for the Central Universities (YWF-15-GJSYS-018).

\section{References}

[1] Wlike I, Kau H P, A numerical investigation of the flow mechanisms in a HPC front stage with axial slots [R]. ASME Paper GT2003-38481, 2003.

[2] Wlike I, Kau H P, A numerical investigation of the influence of casing treatments on the tip leakage flow in a HPC front stage [R]. ASME Paper GT2002-30642, 2002.

[3] Hoffman W H, Ballman J. Some aspects of tip vortex behavior in a transonic turbocompressor [J]. ISABE 2003-1223, 2003.

[4] Hoffman W H, Ballman J. Tip clearance vortex development and shock-vortex-interaction in a transonic compressor ro-tor[R].AIAA-2002-0083, 2002.

[5] Koch C C, Smith L H. Experimental evaluation of outer case blowing or bleeding of a single stage axial flow compressor, part II-performance of plain casing insert configuration with undistorted inlet flow and boundary layer trip [R]. NASA CR-54588, 1968.

[6] Koch C C, Smith L H. Experimental evaluation of outer case blowing or bleeding of a single stage axial flow compressor, Part III-performance of blowing insert configuration No.1 [R]. NASA CR-54589, 1968.

[7] Koch C C, Smith L H. Experimental evaluation of outer case blowing or bleeding of a single stage axial flow compressor, Part IV-performance of plain bleeding insert configuration No.3 [R]. NASA CR-54590, 1968.

[8] Koch C C, Smith L H. Experimental evaluation of outer case blowing or bleeding of a single stage axial flow compressor, Part V-performance of plain casing insert configuration with distorted inlet flow [R]. NASA CR-54591, 1969.

[9] Koch C C, Smith L H. Experimental evaluation of outer case blowing or bleeding of a single stage axial flow compressor, Part VI-final report [R]. NASA CR-54592, 1970.

[10] Greitzer, E M., Nikkanen, J P, Haddad, D E, et al. A Fundamental Criterion for the Application of Rotor Casing Treatment [J]. Journal of Fluids Engineering, 1979, 101(4): 237-243.

[11] Osborn W M, Lenis G W, Heidelberg L J. Effect of several porous casing treatments on stall limit and on overall performance of an axial flow compressor rotor [R]. NASA TND-6537, 1971. 
[12] Prince D C, Wisler D C, Hilvers D E. A study of casing treatment stall margin improvement phenomena [R]. ASME 75-GT-60, 1975.

[13] Müller M W, Biela C, Schiffer H P, et al. Interaction of rotor and casing treatment flow in an axial single-stage transonic compressor with circumferential grooves [R]. ASME Paper GT2008-50135, 2008.

[14] LI Xiangjun, CHU Wuli, ZHANG Haoguang. Stabilizing mechanism of shallow circumferential grooves on a high loaded transonic axial-flow compressor [J]. Journal of Propul-sion Technology, 2013, 34(5):629-637.

[15] Lu Xingen, Chu Wuli, Zhu Junqiang. Mechanism of the Interaction between casing treatment and tip leakage flow in a sub-sonic axial compressor [R]. ASME Paper GT2006-90077,2006.

[16] Hathaway M D. Self-recirculating Casing Treatment Concept for enhanced compressor performance [R]. ASME Paper GT2002-30368, 2002.

[17]LI Ling, YU Qing, LU Yajun. Experimental research of casing treatment on single-stage transonic fan [J]. Journal of Pro-pulsion Technology, 2000, 21(3):49-52.

[18]ZHANG Yandong, LU Yajun. An experimental investigation on arc skewed slot casing treatment [J]. Journal of Aerospace Power, 1998(03). 